\title{
QUASISPECIES DEVELOPMENT BY HIGH FREQUENCY RNA RECOMBINATION DURING MHV PERSISTENCE
}

\author{
C. L. Rowe, ${ }^{1}$ S. C. Baker, ${ }^{1}$ M. J. Nathan, ${ }^{2}$ J.-Y. Sgro, ${ }^{3}$ A. C. Palmenberg,,${ }^{3,4}$ \\ and J. O. Fleming ${ }^{2}$ \\ 'Department of Microbiology and Immunology and Molecular Biology \\ Program \\ Loyola University of Chicago \\ Stritch School of Medicine \\ Maywood, Illinois 60153 \\ ${ }^{2}$ Departments of Neurology and Medical Microbiology \\ University of Wisconsin and William S. Middleton Veterans Hospital \\ Madison, Wisconsin \\ ${ }^{3}$ Institute for Molecular Virology \\ ${ }^{4}$ Department of Animal Health and Biomedical Science \\ University of Wisconsin \\ Madison, Wisconsin
}

\section{ABSTRACT}

Recent studies suggest that infectious viruses and particularly persisting viral RNAs often exist as diverse populations or "quasispecies". We have developed an approach to characterize populations of the murine coronavirus mouse hepatitis virus (MHV) generated during persistent infection which has allowed us to begin to address the role of the viral quasispecies in MHV pathogenesis. We analyzed the population of persisting viral RNAs using reverse-transcription polymerase chain reaction amplification (RT-PCR) of the S1 "hypervariable" region of the spike gene followed by differential colony hybridization to identify spike deletion variants (SDVs) from acute and persistently infected mice. Sequence analysis revealed that mice with the most severe chronic paralysis harbored the most complex quasispecies. Mapping of the SDVs to the predicted RNA secondary structure of the spike RNA revealed that an isolated stem loop structure is frequently deleted. Overall, these results are consistent with high frequency recombination at sites of RNA 
secondary structure contributing to expansion of the viral quasispecies and persisting viral pathogenesis.

\section{INTRODUCTION}

Viruses with RNA genomes rapidly adapt to changing environments. This is because they exist as a "quasispecies" of replicating RNAs (Novella et al., 1995). Two major mechanisms contribute to the viral quasispecies: mutation and RNA recombination. Mutations are rapidly incorporated into the population because RNA viruses have no known proof-reading ability, and therefore are unable to correct random mutations introduced into their viral genome. In addition, RNA viruses have been shown to undergo RNA recombination which may radically alter or delete portions of the RNA virus genome. Copychoice RNA recombination, as described for poliovirus, occurs when partially synthesized RNAs dissociate from one template and then re-join the same or another template at either the same site (homologous recombination) or a different site (aberrant-homologous or non-homologous recombination) (Lai 1992). Both mutation and RNA recombination contribute to quasispecies expansion during persistent infection of MHV and may significantly contribute to viral pathogenesis.

A number of investigators have identified infectious MHV variants during the subacute stage of disease which harbor deletions in the S1 "hypervariable region" (nt 1200-1800) (reviewed in Rowe et al, 1997a). However, the significance of these variants in chronic disease is complex and requires further investigation. During the chronic stage of disease there is a significant reduction in infectious virus, however persisting viral RNA can be detected by RT-PCR amplification (Adami et al., 1995). We wanted to know if genetic changes in the viral RNA might contribute to persistence and chronic disease. To begin to understand the significance of genetic changes in the spike gene, we monitored the genetic changes in the S1 hypervariable region and determined if these genetic changes were associated with more chronic and severe disease.

\section{MATERIALS AND METHODS}

As reported in Rowe et al. 1997a and 1997b, C57BL/6 mice were inoculated intracerebrally with $10^{3}$ p.f.u. MHV-JHM 2.2-V-1 and periodically monitored for clinical signs of demyelination. Mice were sacrificed at 4, 42 and 100 days post-inoculation (PI), and total RNA was isolated from the brains and spinal cords of individual mice. An $848 \mathrm{bp} \mathrm{S1}$ region of the spike gene was amplified by RT-PCR from total RNA with primers S1-1 and S1-2. To identify individual isolates containing SDVs, the S1 products were cloned into pGEM-T (Promega, Madison, WI), transformed into competent E. coli and analyzed by differential colony hybridization using an 837 bp wild-type (WT) probe and small 40-80 bp probes to the core $(C)$ of the hypervariable region and to flanking regions $A, B$ and $D$. Clones which hybridized with the wild-type probe but did not hybridize with probes A-D were sequenced by the sanger dideoxy chain termination method.

Using MFOLD version 2.2, the RNA secondary structure of the 4,139 nt MHV-JHM 2.2-V-1 spike RNA [(accession \# D10235) according to Taguchi et al. (Taguchi et al., 1992) with mutations incorporated according to Wang et al. (Wang et al., 1992)] was folded (Rowe et al., 1997b). 


\section{RESULTS AND DISCUSSION}

We were interested in determining the extent of the diversity in the population of persisting MHV RNAs. We also wanted to determine if changes in the viral RNA were associated with chronic disease and what mechanisms might be responsible for putative changes in the persisting viral RNA. To address these questions, we amplified the spike "hypervariable" region from acute and persistently infected mice (Figure 1). We identified SDVs using differential colony hybridization with S1 subdomain probes (A-D) and a full length probe (WT) (Rowe et al., 1997b). As reported (Rowe et al., 1997a, b), we identified 25 different patterns of SDVs. Interestingly, we found distinct quasispecies populations in the CNS of individual mice. For example mouse $42-8$, which recovered from paralysis, had no detectable SDVs in the brain, only wild-type sequences and only a very low level of a single type of SDV was detected in the spinal cord (Figure 1B). In contrast mouse $100-1$, which displayed chronic severe paralysis, harbored a higher frequency of SDVs both in the brain and the spinal cord and a more diverse quasispecies. In fact, our screening identified four distinct types of SDVs in the spinal cord of mouse 100-1 alone. We consistently found this pattern, where mice with the highest frequency and most diverse quasispecies had the most severe disease (Rowe et al, 1997a). These observations are con-

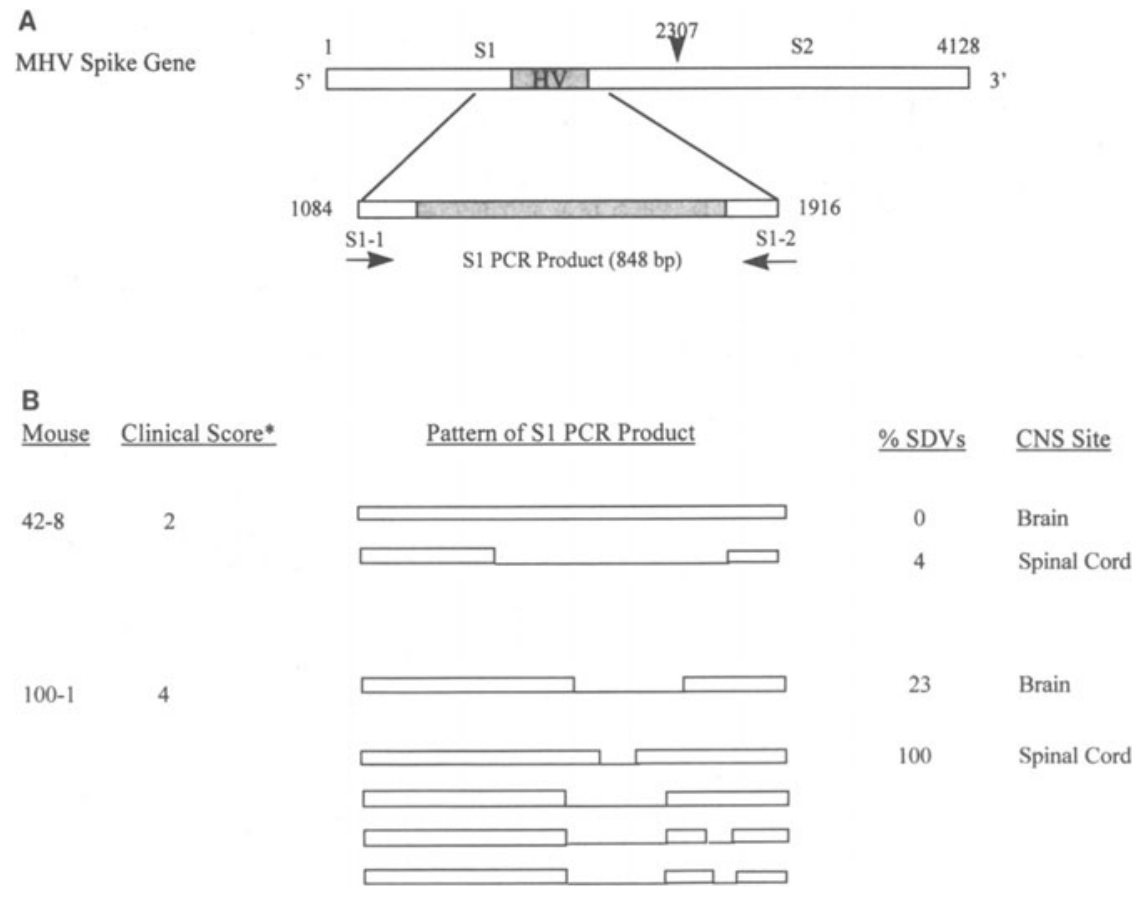

Figure 1. A) Schematic diagram of the position of the S1 primers used to amplify SDVs from the brain and spinal cord of acute and persistently infected mice. B) Examples of SDV patterns identified in mice with different clinical outcomes. Mice were monitored and scored on a scale from 0 (normal) to 4 (severe paralysis) every four days after inoculation. ${ }^{*}$ Clinical score at day 24 . 
A

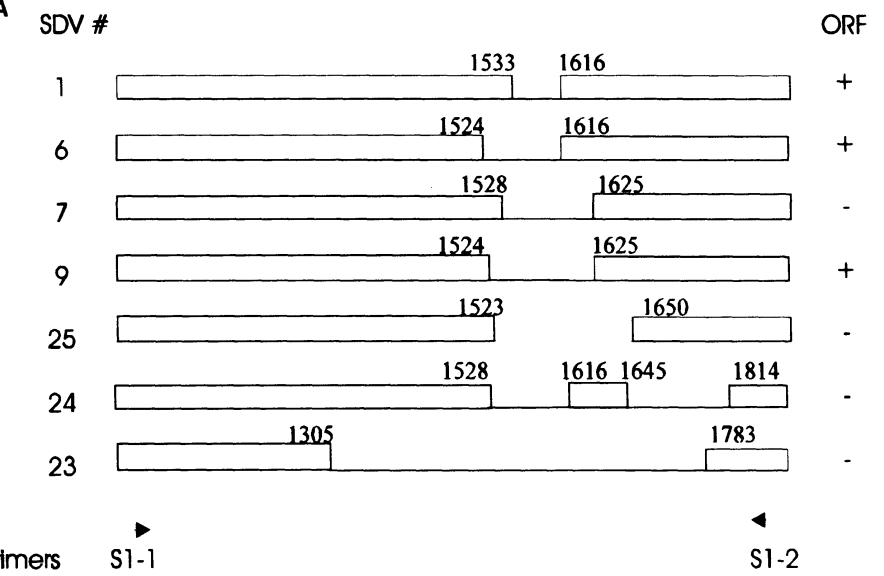

Figure 2A. Schematic diagram of the SDV patterns identified by differential colony hybridization with probes A, $\mathrm{B}, \mathrm{C}, \mathrm{D}$ and WT.

sistent with those seen in chronic infections with hepatitis $C$ virus (Martell et al., 1992) and measles virus (Baczko et al., 1993). Thus, by studying the dynamics of MHV persistence in mice we may be better able to understand how persistent viral infections may contribute to chronic diseases such as MS.

We also wanted to determine how MHV quasispecies arise. RNA secondary structure has been shown to be important in directing recombination in a number of RNA viruses such as tomato bushy stunt virus (TBSV) and turnip crinkle virus (TCV) (White et al, 1994; Cascone et al., 1993). In fact, Simon and colleagues has shown that single base mutations that disrupt a stem loop eliminate recombination events in TCV (Cascone et al., 1993). To analyze the role of RNA secondary structure in the generation of coronavirus SDVs, we mapped a subset of the SDV patterns onto the MHV $4 \mathrm{~kb}$ spike RNA secondary predicted by the computer program MFOLD (Figure 2).

We noted that the SDVs frequently map to the base of an isolated stem loop structure, suggesting that this region may be prone to RNA recombination. In addition, the SDV deletion endpoints were frequently proximal to one another, suggesting that these SDVs may have been generated by an intramolecular recombination event in which the polymerase encounters the stem loop and instead of reading through it, simply jumps across to a nearby site, thereby deleting the stem. By modeling the MHV spike RNA and mapping of spike deletion variants we identified a putative RNA secondary structure which may play an important role in the high frequency generation of SDVs during persistence.

Figure 3 highlights factors which may affect MHV quasispecies populations. Potential selective pressures include: 1) altered binding of variants to host specific receptors, 2) replication advantages of variants, 3 ) altered recognition of variants by cytotoxic $\mathrm{T}$ lymphocytes, 4) reduced capacity for fusion of variants, and 5) reduced cytopathic effects of variants. Interestingly, a spike protein epitope (Db-restricted; a.a 510-518) recognized by CD8+ cytotoxic T lymphocytes (CTLs) is deleted in all of the SDVs that we identified. CTL escape may be one way that the virus persists and causes disease. However, we also identified wild-type epitopes which persisted in some mice, suggesting that deletion of the CTL epitope is not the only way that MHV persists. 


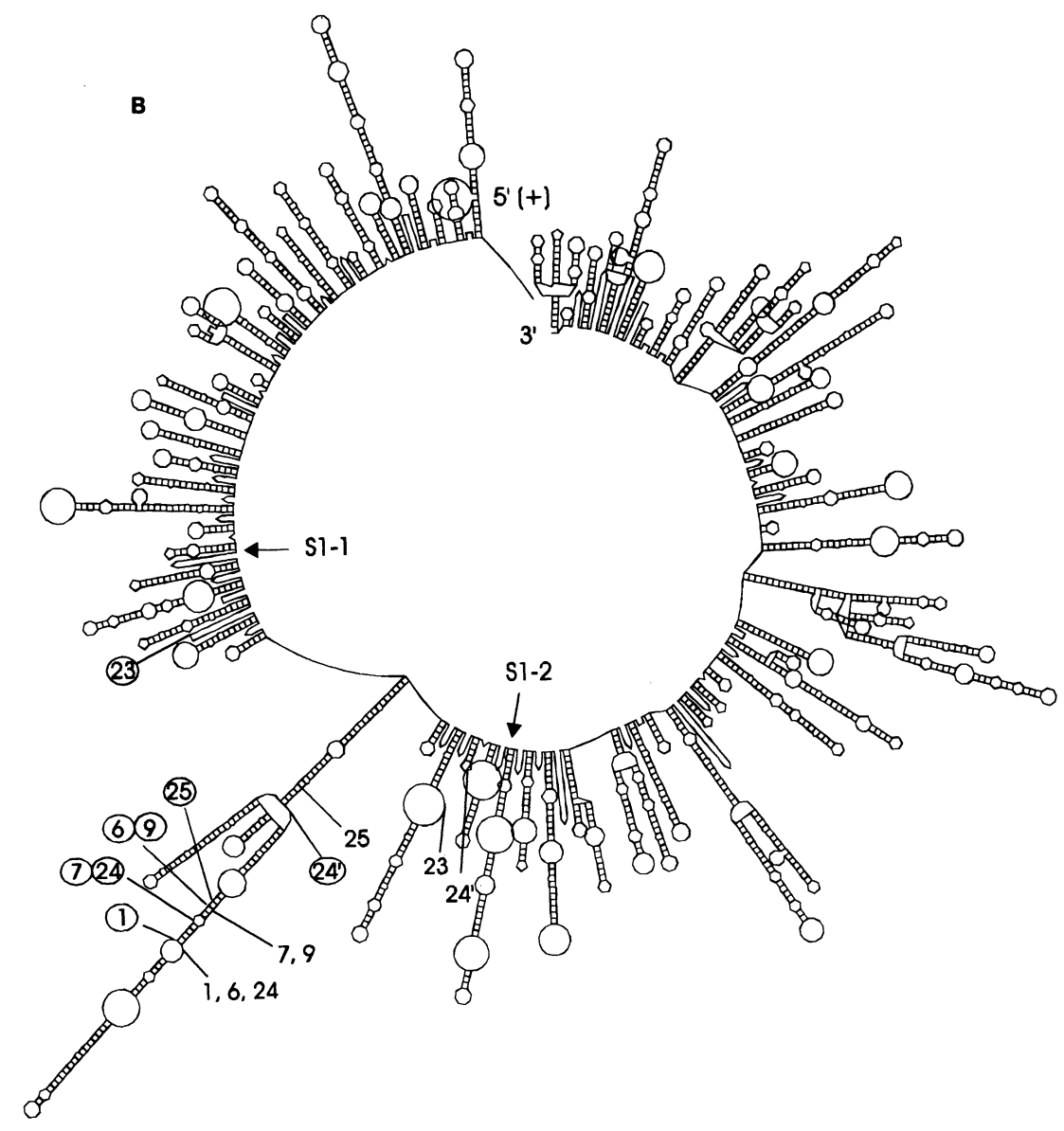

Figure 2B. Mapping of SDV patterns from (A) to the predicted RNA secondary structure of the MHV spike RNA. The left (circled) and right crossover sites of the SDVs are indicated. 


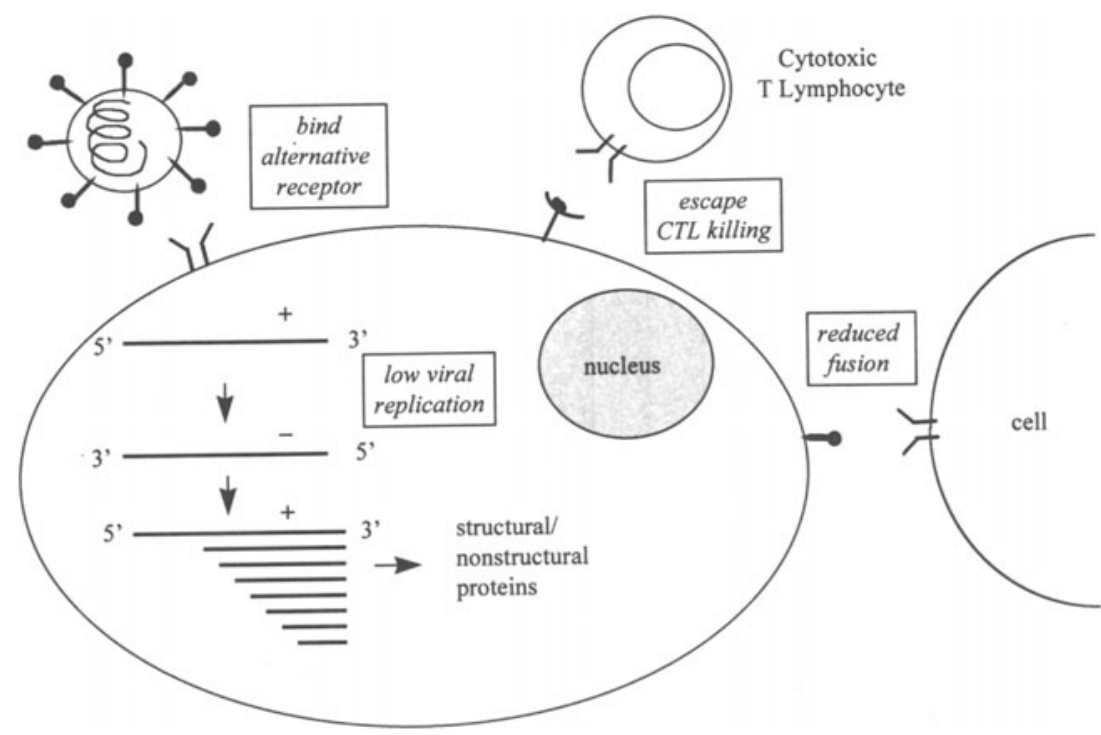

Figure 3. Factors which may contribute to selection of persisting virus or viral RNAs.

\section{REFERENCES}

Adami, C., J. Pooley, J. Glomb, E. Stecker, F. Fazal, J. O. Fleming, and S. C. Baker, 1995, Evolution of mouse hepatitis virus (MHV) during chronic infection: quasispecies nature of the persisting MHV RNA, Virology 209:337-346.

Baczko, K., J. Lampe, U. G. Liebert, U. Brinckmann, V. ter Meulen, I. Pardowitz, H. Budka, S. L. Cosby, S. Isserte, and B. K. Rima, 1993, Clonal expansion of hypermutated measles virus in a SSPE brain, Virology 197: 188-195.

Bergmann, C. C., Q. Yao, M. Lin, and S. A. Stohlman, 1996, The JHM strain of mouse hepatitis virus induces a spike protein-specific Db-restricted cytotoxic T cell response, J. Gen. Virol. 77:315-325.

Cascone, P. J., T. F. Haydar, and A. E. Simon, 1993, Sequences and structures required for recombination between virus-associated RNAs, Science 260:801-805.

Castro, R. F., and S. Perlman, 1995, CD8+ T-cell epitopes within the surface glycoprotein of a neurotropic coronavirus and correlation with pathogenicitiy, J. Virol. 69:8127-8131.

Lai, M. M., 1992, RNA recombination in animal and plant viruses, Microbiol. Rev. 556:61-79.

Martell, M., J. I. Esteban, J. Quer, J. Genesca, A. Weiner, R. Esteban, J. Guardia, and J. Gomez., 1992, Hepatitis C virus $(\mathrm{HCV})$ circulates as a population of different but closely related genomes: quasispecies nature of HCV genome distribution, J. Virol. 66:2547-2556.

Novella, I. S., E. Domingo, and J. J. Holland, 1995, Rapid viral quasispecies evolution - implications for vaccine and drug strategies, [Review]. Molecular Medicine Today 1:248-253.

Parker, S. E., T. M. Gallagher, and M. J. Buchmeier, 1989, Sequence analysis reveals extensive polymorphism and evidence of deletions within the E2 glycoprotein gene of several strains of murine hepatitis virus, Virology 173:664-673.

Rowe, C. L., S. C. Baker, M. J. Nathan, and J. O. Fleming, 1997a, Evolution of Mouse Hepatitis Virus: Detection and Characterization of Spike Deletion Variants During Persistent Infection, J. Virol. 71:2959-2969.

Rowe, C. L., J. O. Fleming, J.-Y. Sgro, A. C. Palmenberg and S. C. Baker, 1997b, Generation of coronavirus spike deletion variants (SDVs) by high frequency recombination at regions of predicted RNA secondary structure, J. Virol., in press. 
Taguchi, F., T. Ikeda, and H. Shida, 1992, Molecular cloning and expression of a spike protein of neurovirulent murine coronavirus JHMV variant cl-2 [published erratum appears in J Gen Virol 1992 Oct; 73(Pt10):2767], J. Gen. Virol. 73:1065-1072.

Wang, F. I., J. O. Fleming, and M. M. Lai, 1992, Sequence analysis of the spike protein gene of murine coronavirus variants: study of genetic sites affecting neuropathogenicity, Virology 186:742-749.

White, K. A., and T. J. Morris, 1995, RNA determinants of junction site selection in RNA virus recombinants and defective interfering RNAs. RNA 1:1029-1040. 